\title{
AUTOMATIC ESTIMATION OF ALTITUDE OF THE REFERENCES POINTS FOR AERIAL AUTONOMOUS NAVIGATION USING AERIAL PHOTOGRAPHS AND CHARACTERISTIC POINTS
}

\author{
M.A. P. Domiciano ${ }^{1}$, E. H. Shiguemori ${ }^{1}$, L. A. V. Dias $^{2}$ \\ ${ }^{1}$ Department of Structural and Geotechnical Engineering, Institute for Advanced Studies \\ (elcio@ieav.cta.br or pizani@ieav.cta.br) \\ ${ }^{2}$ Department of Computer Engineering, Brazilian Aeronautics Institute of Technology
}

\begin{abstract}
The use of computer vision techniques to solve problems related to estimation of position has increased in recent decades due to rapid technological and computing development. This paper presents an approach to obtain position information of an Unmanned Aerial Vehicle (UAV) based on aerial images. The problem addressed is to allow a UAV to be located above ground. The considered vehicle has an inertial navigation system (INS) and receives no assistance of a satellite navigation system. Due to the inherent errors of the INS that cause disorientation of the UAV, an image-tracking system is proposed. The proposed methodology has been to build, in real-time and embedded, a Digital Elevation Model of reference points (DEM-R) to allow a vehicle to locate in the ground based on the comparison between this and pre-existing model. In order to achieve this objective it is necessary to identify similar points in sequential images and calculate the altitude of these points. This enables build the DEM-R. We used the SIFT techniques and Zernike moments to identify similar points. We used aerial imagery of the city of Sao Jose dos Campos - Sao Paulo - Brazil, with longitudinal coverage of 60\%. The obtained results show that the methodology is viable to the proposed problem, autonomous navigation of UAVs.
\end{abstract}

Keywords: Digital Elevation Model, Images Processing, Photogrammetry, Real Time.

\section{INTRODUCTION}

The advancement of technology and development of Computer Science have allowed the use of images to solve applications that require answers in a short period of time.

One application that has benefited from this development was aerial autonomous navigation based on images.

The UAV stands out, especially in missions where the presence of a crew on board may be a limiting factor. One of the current challenges is to provide a UAV capability of autonomous navigation with the least possible dependence on satellite navigation systems (Global Navigation Satellite System). 
Navigation of a UAV may be accomplished by different ways, including, in a controlled manner or autonomously. At first, the aircraft is controlled remotely via a control station. In autonomous navigation in an embedded system captures the UAV flight information, to re-plan your navigation automatically.

In autonomous navigation, a navigation module should be able to ensure compliance with a pre-planned route, safely and efficiently.

This paper addresses a possibility to get the aircraft's position in a given time based on the construction of a digital elevation model (DEM) from the images in order to compare it with a pre-existing [1], [2], [3].

\section{PROBLEM}

In [4] is cited as the first operational UAV was A.M. Low's "aerial target" of 1916, and that the scientist Hugo Gernsback had already realized the advantages of unmanned vehicle control link radio and television in 1924, although the technology existing at that time proved to be inadequate.

According to [5] the UAVs have been often utilized on missions that are too "dull, dirty, or dangerous" for manned aircraft.

In autonomous navigation, it is important a accurate extraction of the navigation parameters to airplane, such as position and velocity. Using these parameters one cans adjust the speed and direction to the desired destination [6].

One possibility to obtain the position of the aircraft in a given time is based on construction, real-time and embedded, of a digital elevation model of references (DEM-R) from the images obtained during the flight of a UAV and compare it with a model pre-existing [1], [2], [7].

\section{METHODOLOGY}

The methodology consisted of the following steps: to find characteristic points in images with the application of the SIFT algorithm [7], make the correspondence between the centers of the images in order to find the misalignment between them.

After that it is necessary to find the starting point of search. Then, it is applied the Zernike Moments (ZM) [8], [9] from this starting point in parts of the images, windows search, find corresponding points, and finally the altitude is estimated.

In order to compare the results was made as follows: to find characteristic points in images with the application of the SIFT algorithm [7], make the correspondence between the centers of the images in order to find the misalignment between them and finding the starting point of search. Then, it is applied a polynomial of fourth degree from this starting point in parts of the images, find corresponding points, and finally the altitude is estimated.

Then the altitude of each point identified in relation to a datum specified has been obtained using the following equation used in photogrammetry [1], [10]. 


$$
h_{a}=H-\frac{B . f}{x_{a}-x^{\prime} a} .
$$

where $H$ is the altitude of flight in relation to a datum, $B$ the air baseline, $f$ the focal distance, $x_{\mathrm{a}}$ the distance from the point " $\mathrm{A}$ " to the center of the first image and $x_{\mathrm{a}}^{\prime}$ the distance from the point "A" to the center point of the second image.

\section{RESULTS}

For preliminary results obtained in the study, it has been employed two aerial photos of the city of Sao Jose dos Campos, Brazil, with overlap of ap-proximately $60 \%$. These images were provided by [11]. The methodology applied in these images obtained altitudes close to the real terrain, indicating that it can be applied in the estimation of position of the UAV through the DEM-R.

The next step is to compare the obtained DEM-R with the pre-existing reference model to estimate the error of the algorithm.

In order to compare the obtained altitudes with the reference ones, it was selected a part of the image that contains the runway of the city.

The criteria for choosing this area were:

- The area is approximately flat;

- To have the DEM of the area;

- The area has a slope;

- Ease of access, and

- Easily identifiable in the image
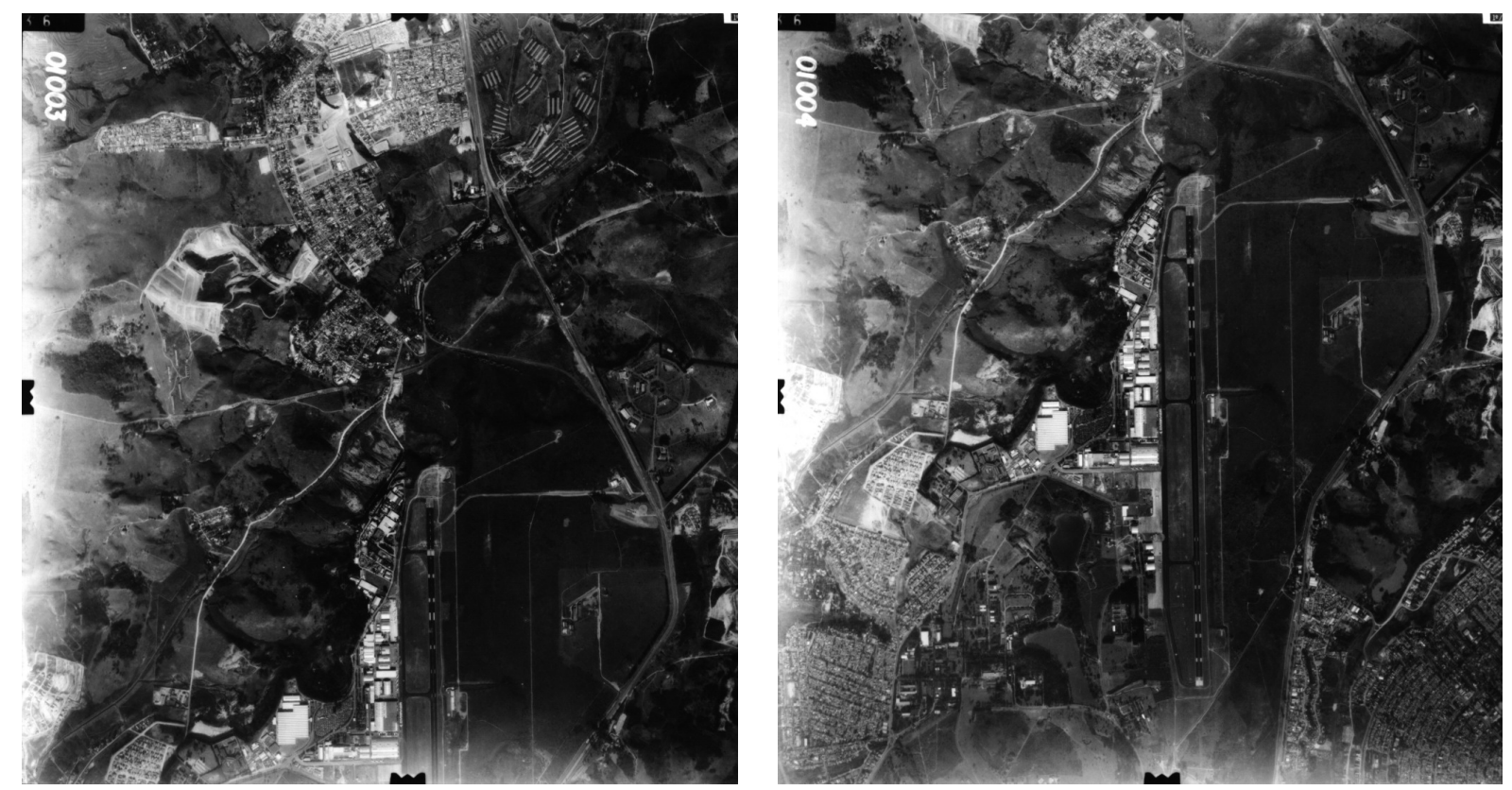

Figure 1 - Sao Jose dos Campos Images 
Figure 1 shows some Sao Jose dos Campos images used in this work.

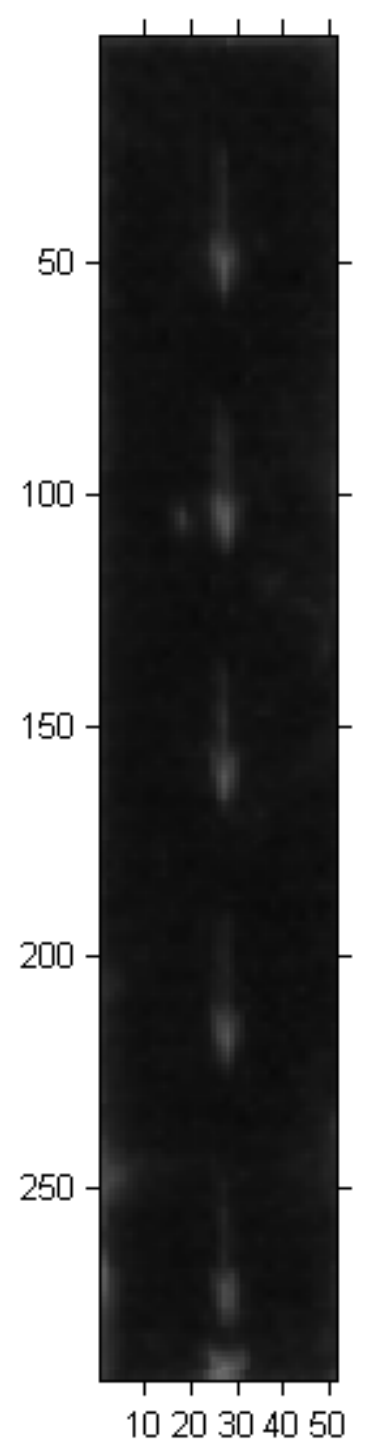

Figure 2 - Part of the Runway of Sao Jose dos Campos

Figure 2 shows the area of the runway utilized in tests.

\subsection{Calculated DEM_R_ZM}

It was applied Zernike moments on the area corresponding to the runway and performed a subsequent search to locate the corresponding points between two images of the runway by applying the Zernike Moments. After that, it was calculated the altitudes of these points as shown in Figure 3 (Calculated DEM_R_ZM).

The results obtained are similar to the altitude of the reference DEM-R, as can be seen in Figure 3.

The resolution of the reference DEM-R is approximately $5 \mathrm{~m}$ while the resolution of the images used is approximately $0.858 \mathrm{~m}$, because the resolutions are not equal or propor- 
tional and using six pixels in an image to one in the DEM-R, each 600 pixels in the image correspond to 103 pixels in the DEM-R and not 100, thus there may be a mismatch of altitudes.
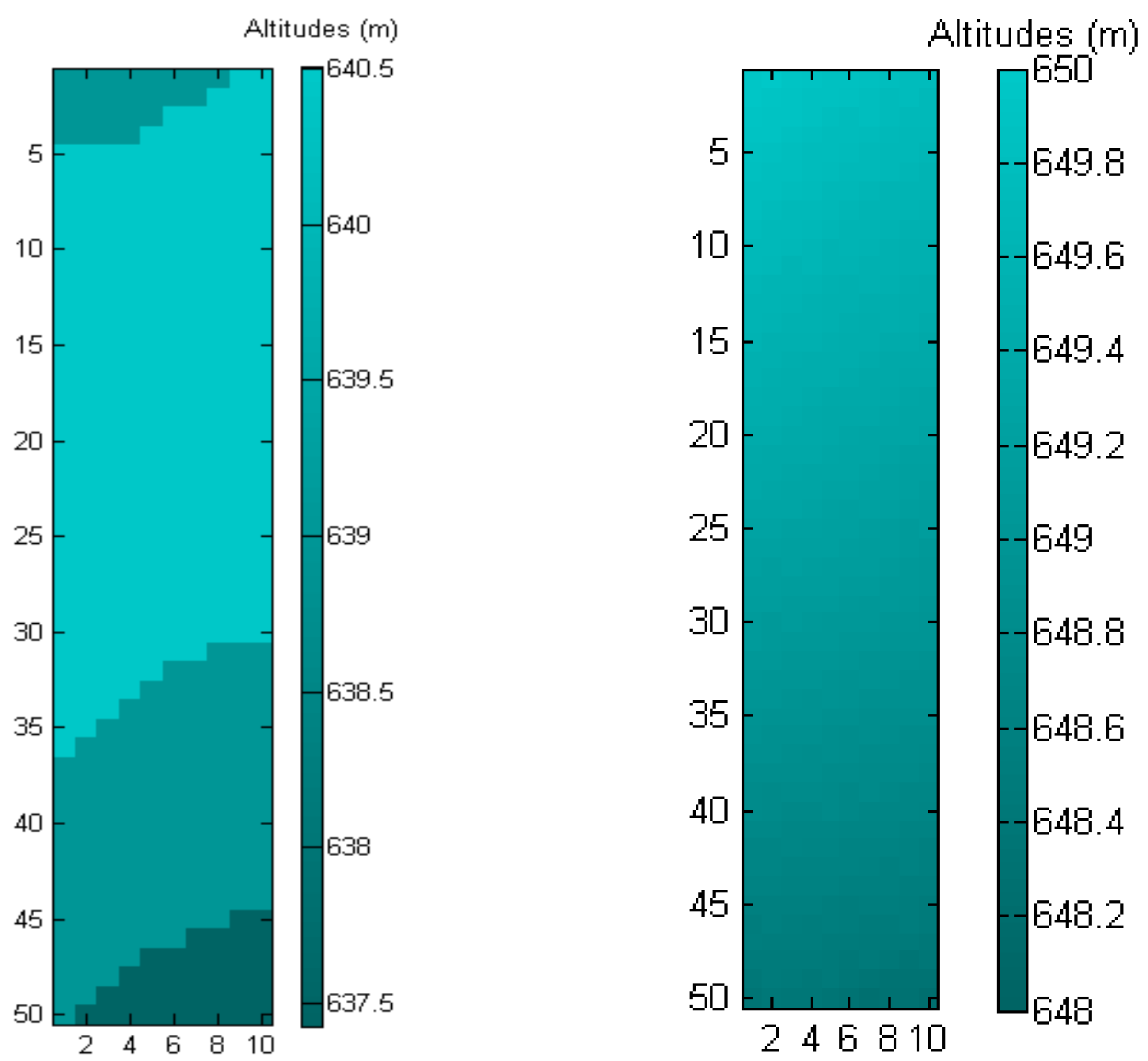

Figure 3 - Calculated DEM-R_ZM and reference DEM-R

The spatial resolution along with the fact that the area is flat with a slight slope, contributes to small displacements of points, providing similar altitudes in the range analyzed.

Comparing the reference DEM-R with calculated DEM-R_ZM it was obtained for the maximum altitude $649.9690 \mathrm{~m}$ for the reference DEM-R and $640.5026 \mathrm{~m}$ for the calculated DEM-R.

As far as difference between the maximum altitude of the reference DEM-R and the calculated DEM-R_ZM it was obtained $9.488 \mathrm{~m}(1.46 \%)$.

Comparing the reference DEM-R with calculated DEM-R_ZM it was obtained for the minimum altitude $648.2220 \mathrm{~m}$ for the reference DEM-R and $637.4209 \mathrm{~m}$ for the calculated DEM-R.

As far as difference between the minimum altitude of the reference DEM-R and the calculated DEM-R_ZM it was obtained $10.8011 \mathrm{~m}(1.67 \%)$.

The minimum difference for altitudes on the calculated DEM-R_ZM was $3.0817 \mathrm{~m}$, and for the reference DEM-R was $1.747 \mathrm{~m}$. 
The average absolute difference among the altitudes on reference and the calculated DEM-R was $9.3885 \mathrm{~m}$, been this result is equivalent to an average error of $1.45 \%$.

\subsection{Calculated DEM_R_4P}

By way of comparison the same part of the image was applied SIFT algorithm to find points corresponding initial these points are not regularly arranged. After that, it was calculated the coefficients of a polynomial of the fourth degree and thus obtain the corresponding points on each image to calculate the DEM_R_4P (Figure 4).

The results obtained are similar to the altitude of the reference DEM-R, as can be seen in Figure 4.

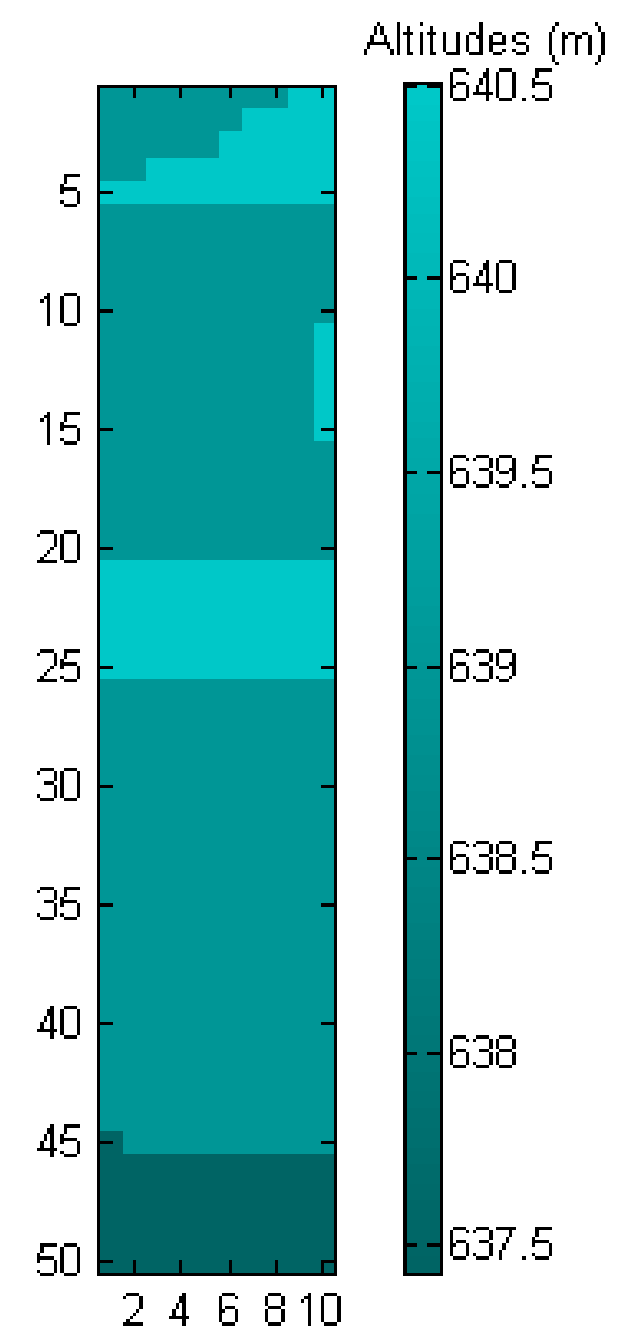

Figure 4 - Calculated DEM-R_4P and reference

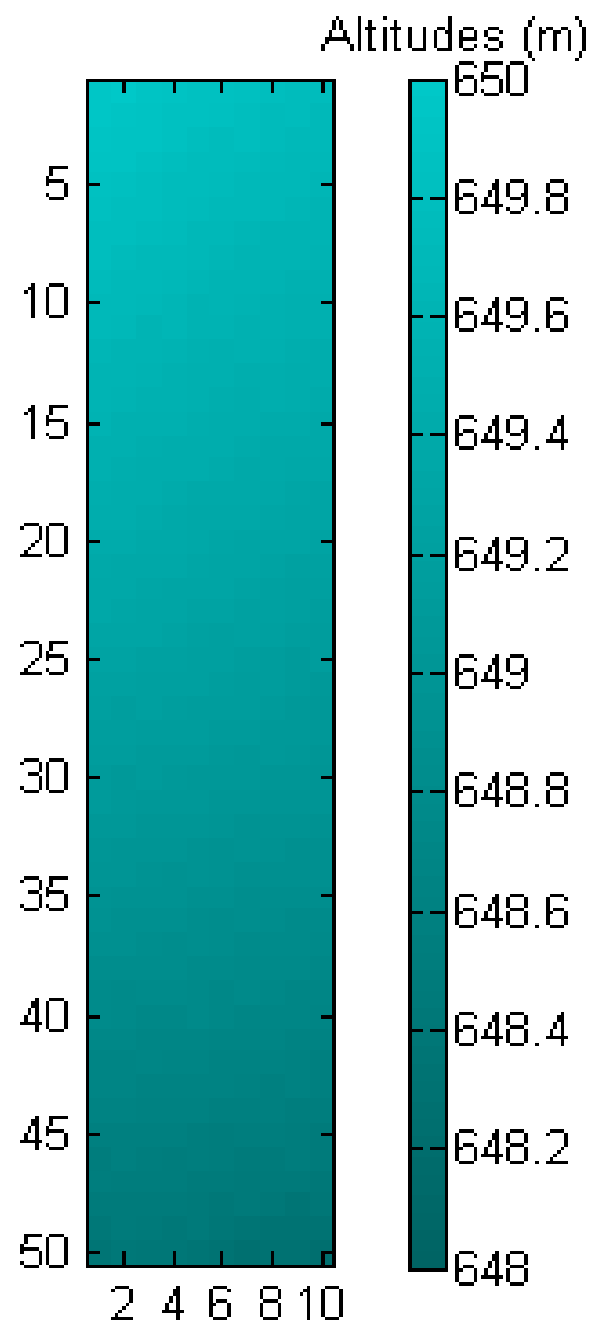

DEM-R

The spatial resolution along with the fact that the area is flat with a slight slope, contributes to small displacements of points, providing similar altitudes in the range analyzed.

Comparing the reference DEM-R with calculated DEM-R_4P it was obtained for the maximum altitude $649.9690 \mathrm{~m}$ for the reference DEM-R and $640.5026 \mathrm{~m}$ for the calculated DEM-R. 
As far as difference between the maximum altitude of the reference DEM-R and the calculated DEM-R_4P it was obtained $9.488 \mathrm{~m}(1.46 \%)$.

Comparing the reference DEM-R with calculated DEM-R_4P it was obtained for the minimum altitude $648.2220 \mathrm{~m}$ for the reference DEM-R and $637.4209 \mathrm{~m}$ for the calculated DEM-R.

As far as difference between the minimum altitude of the reference DEM-R and the calculated DEM-R_4P it was obtained $10.8011 \mathrm{~m}(1.67 \%)$.

The minimum difference for altitudes on the calculated DEM-R_4P was $3.0817 \mathrm{~m}$, and for the reference DEM-R was $1.747 \mathrm{~m}$.

The average absolute difference among the altitudes on reference and the calculated DEM-R was $10.0939 \mathrm{~m}$, been this result is equivalent to an average error of $1.56 \%$.

The imprecision of the measurements of height, the median plane, flight altitude, and air baseline are sources of error for automatic determination of altitudes.

\section{CONCLUSION}

This paper presented a methodology for obtaining elevations of terrain points using aerial photographs and their characteristics. The results are promising, however, work is still under way and different methodologies and case studies will be discussed with the aim of application in autonomous UAV navigation.

\section{REFERENCES}

[1] M. A. P. Domiciano, R. A. P. Vitalli, E. H. Shiguemori, L. A. V. Dias, "Estudo da Influência do Tamanho Real do CCD para o Cálculo do DEM”, SERFA, Nov. 2008.

[2] H. Schultz, “Terrain Reconstruction from Widely Separated Images”. Proc. SPIE, vol. 2486, pp. 113-123, Apr. 1995.

[3] H. Schultz, A. R. Hanson, E. M. Riseman, F. Stolle, Z. Zhu, C. D. Hayward, D. Slaymaker, "A System for Real-time Generation of Geo-referenced Terrain Models". SPIE Enabling Technologies for Law Enforcement, 2000.

[4] G. Simons. Drone Diplomacy. The Link. New York, Americans for Middle East Understanding, Inc. 44: 16, 2001.

[5] M. Kane. Autonomous Systems and Future Capability, Bae Systems, 2007.

[6] D. G. SIM; R. H. PARK. Localization Based on DEM Matching Using Multiple Aerial Image Pairs. IEEE Transactions on Image Processing [S.I.], Vol. 11, no 1, p. 52-55, Jan. 2002, 2002.

[7] D.G. Lowe, "Distintive image features from scale-invariant keypoints", International Journal of Computer Vision, vol. 60, no. 2, pp 91-110, 2004.

[8] A. Khotanzad; Y. H. Hong. "Invariant Image Recognition by Zernike Moments." IEEE Transactions on Pattern Analysis and Machine Intelligence 12 (05): 09, 1990.

[9] Z. Chen; S.-K. Sun. "A Zernike Moment Phase-Based Descriptor for Local Image Representation and Matching." IEEE TRANSACTIONS ON IMAGE PROCESSING 19(1): 15, 2010. 
[10] P. R. Wolf. Elements of Photogrammetry, McGraw-Hill. unique: 628, 1983.

[11] $1 \% 6^{\circ}$ Grupo de Aviação. Fotografias Aéreas de São José dos Campos. Recife, Brazil, Brazilian Air Force, 1999. 